\title{
Solitary Cerebral Metastases vs. High-grade Gliomas: Usefulness of Two MRI Signs in the Differential Diagnosis
}

\author{
CARMINE FRANCO MUCCIO ${ }^{1}$, ENRICO TEDESCHI ${ }^{2}$, LORENZO UGGA $^{2}$, \\ RENATO CUOCOLO ${ }^{2}$, GENNARO ESPOSITO ${ }^{1}$ and FERDINANDO CARANCI ${ }^{3}$ \\ ${ }^{1}$ Unit of Neuroradiology, AO "Gaetano Rummo", Benevento, Italy; \\ ${ }^{2}$ Department of Advanced Biomedical Sciences, University of Naples "Federico II", Naples, Italy; \\ ${ }^{3}$ Department of Medicine and Health Sciences "V. Tiberio", University of Molise, Campobasso, Italy
}

\begin{abstract}
Background/Aim: The differentiation between cerebral metastases $(C M)$ and high-grade gliomas $(H G G)$ can be difficult on magnetic resonance imaging (MRI). The aim of this study was to evaluate the usefulness of searching two MRI signs (signal alteration in the adjacent cortex, SAAC, and peripheral rim sign, PRS), in order to distinguish between these entities. Patients and Methods: A total of 61 patients were retrospectively enrolled (28 HGG, 33 CM). Fluid Attenuated Inversion Recovery (FLAIR) sequences were used to assess SAAC and contrast-enhanced T1-weighted sequences for PRS. Results: A positive SAAC sign was present in $61 \%$ of $H G G$, and $12 \%$ of CM. Conversely, in SAAC-negative lesions, PRS was observed in $78 \%$ of $C M$ and in $32 \%$ of $H G G$. Their association had a higher frequency in $H G G$ than in the $C M$ group (21 vs. 3\%). Conclusion: While SAAC is specific for $H G G$ and PRS, in the absence of SAAC, is relatively specific for CMs, their combined presence is highly suggestive of $H G G$.
\end{abstract}

The differential diagnosis between cerebral metastases (CMs) and high-grade gliomas (HGGs) is essential for prognostic evaluation and therapeutic planning. In fact, patients with HGGs more often require neurosurgical resection as an initial approach (1), while CMs are variably treated with either neurosurgical procedure, chemotherapy or immunotherapy, or irradiation, on the basis of primitive tumour and systemic staging (2).

CMs and HGGs are detected as solitary lesions in $50 \%$ and $90 \%$ of the cases, respectively $(3,4)$. When they present as

Correspondence to: Lorenzo Ugga, MD, Department of Advanced Biomedical Sciences, University of Naples "Federico II", Via S. Pansini 5, 80131, Naples, Italy. Tel: +39 0817464646, Fax: +39 0817464646, e-mail: lorenzo.ugga@gmail.com ORCID ID: 00000001-7811-4612

Key Words: MRI, cerebral metastasis, Gadolinium, high grade glioma, differential diagnosis. solitary, solid, contrast-enhancing lesions, the differential diagnosis may, sometimes, be difficult using magnetic resonance imaging (MRI), even with advanced techniques such as diffusion tensor, spectroscopy and perfusion imaging (5-14). Moreover, the routine use of these advanced techniques may be limited by operative conditions, like the availability of optimized acquisition protocols and elaboration software, and by the variable experience of radiologists and neuroradiologists in their interpretation $(15,16)$.

While in some cases clinical history is helpful for formulating the correct diagnosis, neurosurgery and pathology are still often used to reach a definitive diagnosis, as CMs and HGGs may share similar imaging features (7-9). Based on the fact that HGG cells very early infiltrate the surrounding brain tissue (17), the signal alteration in the cortex adjacent to a gadolinium-enhancing lesion (signal alteration in adjacent cortex, SAAC) has been proposed as a useful conventional MRI finding on unenhanced fluid attenuated inversion recovery (FLAIR) T2-weighted (T2w) sequences (17-21).

On the other hand, a recent study on the differential diagnosis between intramedullary spinal cord lesions showed that metastases were more frequently associated with a peripheral rim of higher gadolinium-enhancement, i.e. the peripheral rim sign (PRS), on T1-weighted (T1w) sequences (18). For brain metastases, the presence of this imaging finding has not been assessed, nor has the combination of PRS and SAAC been previously investigated.

Therefore, the purpose of this study was to assess the diagnostic performance of SAAC and PRS, individually and together, in the differential diagnosis between CMs and HGGs presenting as solitary, solid gadolinium-enhancing lesions.

\section{Patients and Methods}

Among the patients referred to our Institution for surgical removal of a primary or secondary brain tumor, 61 adult patients (34 males and 27 females; age range $=37-84$ years) affected by $33 \mathrm{CM}$ (primary cancer: lung $=17$, breast $=9$, colon $=3$, bladder $=1$, kidney $=1$, and uterus cervix=1) and 28 HGG (24 grade IV and 4 grade III) 
were selected. Inclusion criteria required that CMs and HGGs were evident as a single, supratentorial, solid, gadolinium-enhancing lesion, as well as the availability of pathological analysis. Exclusion criteria were: infratentorial CMs and HGGs, the presence of cystic and/or necrotic areas within the lesion, or the inability to obtain an adequate MRI quality, due to excessive movement artefacts.

All MRI studies were performed at the Unit of Neuroradiology of our Institution on the same 1.5 T scanner (Signa EXCITE, General Electric, Milwaukee, WI, USA) provided with 8-channel dedicated coil, using conventional FLAIR-T2w sequences (TE: 80, TR: 8000, inversion time: 2000, frequency: 256, phase: 224, thickness: $5.0 \mathrm{~mm}$, interval: $1.5 \mathrm{~m}$ ) and FLAIR-T1w (TE 9, TR 2100 , TI 700 , frequency 320 , phase 256 , thickness mm 5 , interval $1.5 \mathrm{~mm}$ ) or SPGR-T1w (TE 8.2, Flip Angle $20^{\circ}$, frequency 320 , phase 224, thickness $1.4 \mathrm{~mm}$ ) sequences obtained before and after intravenous (i.v.) administration of gadolinium (Gadobutrol, Gadovist, Bayer-Schering Pharma, Berlin, Germany) at a dose of $0.1 \mathrm{mmol} / \mathrm{kg}$. In particular, FLAIR-T1w was used in 49 cases $(28$ $\mathrm{CM}$ and $21 \mathrm{HGG}$ ) and SPGR-T1w in the remaining 12 (5 CM and 7 HGG). Informed consent was previously obtained from all individual participants included in the study.

Two neuroradiologists (CFM and GE), both with over 10 years of neuroradiological experience, assessed in consensus the presence of SAAC and PRS on unenhanced T2-FLAIR and axial and coronal gadolinium-enhanced T1w MR images, blind to both clinical history and histopathologic diagnosis of the patients.

SAAC was defined as the presence of a non-gadoliniumenhancing signal alteration in the FLAIR T2w image located in the cortex adjacent to a gadolinium-enhancing lesion (16-20) (Figure 1). PRS was defined as a complete or partial (covering $>2 / 3$ of lesion margin), clear-cut peripheral rim of gadolinium-enhancement, more intense than the remaining lesion border (Figure 2).

The chi-square test was used to assess the relationship between the two signs, HGG and CMs. A p-value equal or inferior to 0.05 was considered as statistically significant. Then, based on $2 \times 2$ contingency tables, diagnostic accuracy parameters of SAAC, PRS and their combination were computed. In particular, sensitivity, specificity, positive and negative likelihood ratio, positive and negative predictive value and overall accuracy were calculated. All analyses were conducted with a dedicated software (RStudio http://www.rstudio.com).

\section{Results}

The distribution of SAAC and PRS in HGGs and CMs is shown in Table I, while Table II presents the corresponding measures of diagnostic performance. In particular, SAAC was evident in 17 out of 28 HGGs (60.7\%) and in 4 out of 33 CMs (12.1\%), while PRS was observed in 26 out of 33 CMs $(78.8 \%)$ and in 9 out of 28 HGGs (32.1\%). Two HGGs (7\%) and three CMs (9\%) did not show any SAAC nor PRS.

The Chi-square test showed a significant association between SAAC and HGG $(p<0.001)$ and PRS and CMs $(p<0.001)$. SAAC sensitivity for HGG was $60.7 \%$, with a specificity of $87.9 \%$ (accuracy: $75.4 \%$ ), while PRS sensitivity for CMs was of $78.8 \%$, with a specificity of $67.9 \%$ (accuracy: $73.8 \%$ ). SAAC and PRS were associated in 1 out of $33 \mathrm{CMs}$, and in 6 out of 28 HGGs. In the HGG group, the association
Table I. Prevalence of peripheral rim sign and signal alteration in the adjacent cortex in high-grade gliomas and cerebral metastases.

\begin{tabular}{|c|c|c|c|}
\hline & SAAC & PRS & SAAC and PRS \\
\hline HGGs $(n=28)$ & $17(60.7 \%)$ & $9(32.1 \%)$ & $6(21.4 \%)$ \\
\hline CMs (n=33) & $4(12.1 \%)$ & $26(78.8 \%)$ & $1(3.0 \%)$ \\
\hline
\end{tabular}

SAAC: Signal alteration in the adjacent cortex; PRS: peripheral rim sign; HGGs: high-grade gliomas; CMs: cerebral metastases.

Table II. Measures of diagnostic performance for the evaluated MRI signs in high grade gliomas and metastasis.

\begin{tabular}{lccc}
\hline & SAAC & PRS & SAAC and PRS \\
\hline Sensitivity & $60.7 \%$ & $78.8 \%$ & $21.4 \%$ \\
Specificity & $87.9 \%$ & $67.9 \%$ & $97.0 \%$ \\
Positive likelihood ratio & 5.01 & 2.45 & 7.07 \\
Negative likelihood ratio & 0.45 & 0.31 & 0.81 \\
Positive predictive value & $80.9 \%$ & $74.3 \%$ & $85.7 \%$ \\
Negative predictive value & $72.5 \%$ & $73.1 \%$ & $59.3 \%$ \\
Accuracy & $75.4 \%$ & $73.8 \%$ & $62.3 \%$ \\
\hline
\end{tabular}

SAAC: Signal alteration in the adjacent cortex; PRS: peripheral rim sign.

of SAAC and PRS reached a specificity of $97.0 \%$ and a positive predictive value of $85.7 \%$, but with a sensitivity of $21.4 \%$, and thus a low accuracy $(62.3 \%)$.

\section{Discussion}

We evaluated the possibility of increasing the diagnostic power of conventional MRI in the differential diagnosis between CMs and HGGs and found that SAAC is indicative of HGG, PRS in absence of SAAC- is relatively specific for CMs, and that their combined presence is highly suggestive of HGG.

While the value of SAAC and PRS has been previously assessed in this differential diagnosis (18-20), this is the first study evaluating their combined accuracy. Our results show that in case of a solitary, solid, gadolinium-enhancing lesion of uncertain origin, first the FLAIR T2w images should be closely searched for the SAAC sign, that would indicate the presence of $\mathrm{HGG}$, as they have also been reported previously $(19,20)$. Then, if no SAAC is found, the presence of PRS may be assessed in the contrast-enhanced T1w images, that would point toward the metastatic nature of the lesion.

In unenhanced FLAIR T2w sequences, the signal alteration in the adjacent cortex seems to be relatively more specific, while not particularly sensitive, for HGGs rather than CMs. Tang et al. (19) observed a specificity of $91 \%$ and a sensitivity of $44 \%$, while Muccio et al. (20) reported, 


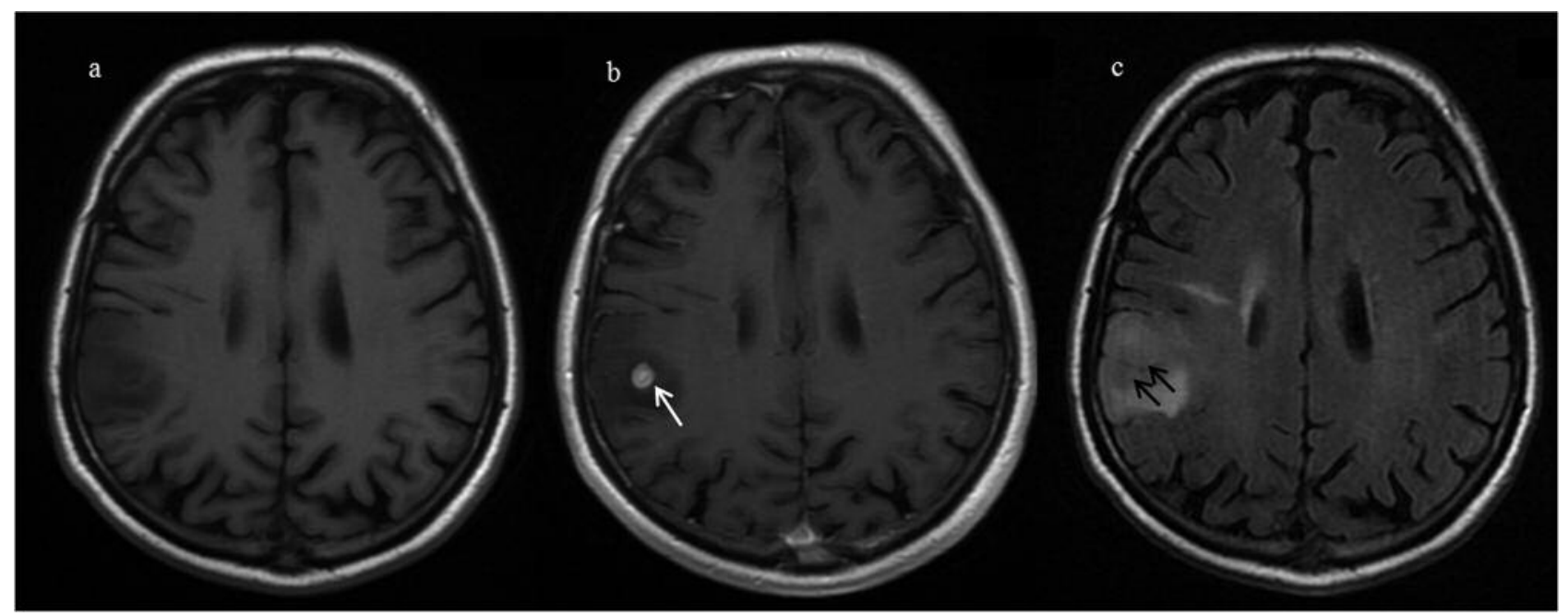

Figure 1. Glioblastoma. Axial FLAIR T1-weighted (a), contrast-enhanced FLAIR T1-weighted $(b)$ and unenhanced FLAIR T2-weighted images. This contrast-enhancing lesion shows a peripheral thin rim of more intense enhancement (white arrow in b), associated with high signal intensity of the adjacent cortex (SAAC) on the unenhanced FLAIR T2-weighted axial image (black arrows in c), i.e. the sign of involvement of the adjacent brain.

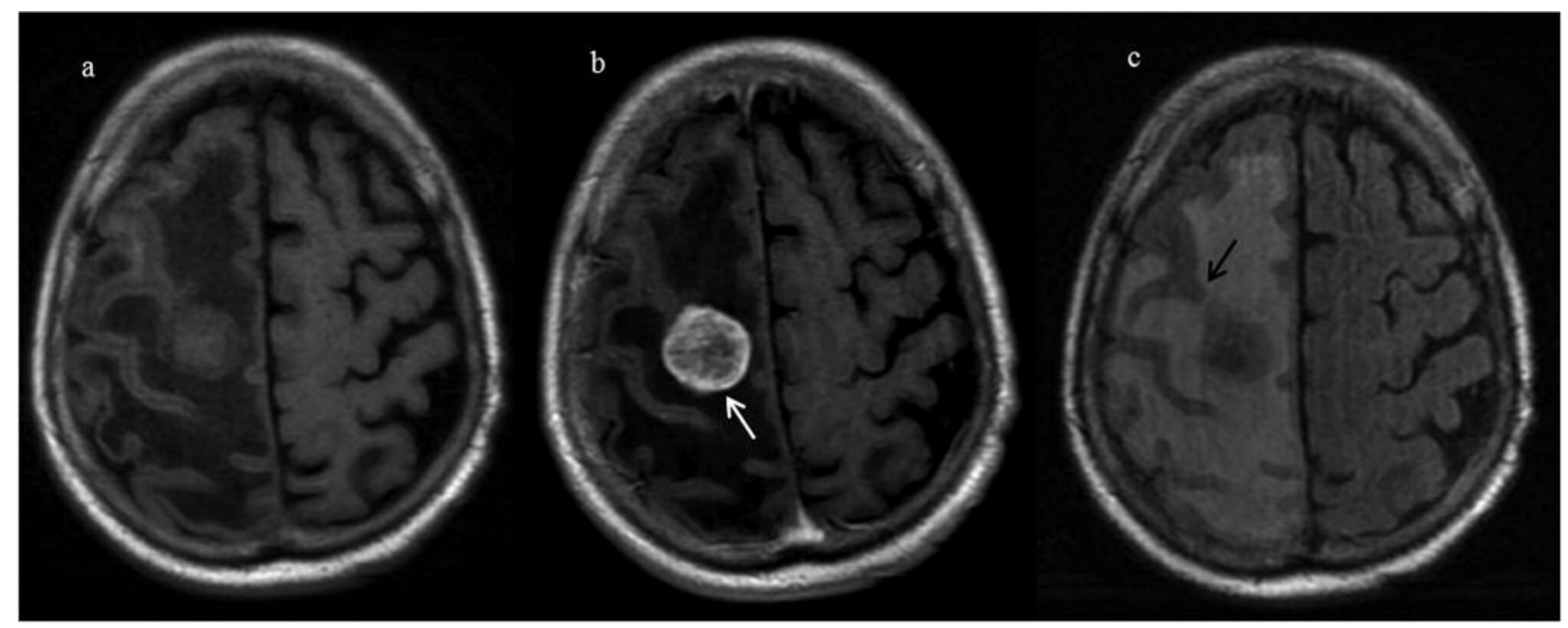

Figure 2. Cerebral metastasis from colon carcinoma. Axial FLAIR T1-weighted (a), contrast-enhanced FLAIR T1-weighted (b) and unenhanced FLAIR T2-weighted images (c). Note the peripheral rim sign (white arrow) on gadolinium-enhanced T1-weighted FLAIR image (b), i.e. a thin rim of more intense enhancement on the outer border of the enhancing lesion. Unenhanced FLAIR T2-weighted axial image (c) does not show signal alteration (black arrow) in the brain cortex adjacent to the area of contrast-enhancement (b).

in a larger population, a specificity of $88 \%$ and a sensitivity of $67,7 \%$. In HGGs, tumour cells infiltrate brain tissue beyond the neoplastic margin (17), thus, gadoliniumenhancement does not coincide with the boundaries of infiltration margin, and should not be used to represent the neoplastic extension. Signal alteration in the unenhanced FLAIR-T2w sequences suggests the presence of tumour infiltration, and is, therefore, more indicative of HGGs than of CMs, even when observed in association with PRS. In fact, vasogenic edema preferentially involves white matter and largely spares gray matter (19); therefore, when a signal intensity change is found within the cortex in proximity to a subcortical lesion, this is likely due to microscopic neoplastic infiltration of the peritumoral edematous region. Even when associated with PRS, SAAC was far more frequent in HGGs $(21 \%)$ than in CMs (3\%).

Recently, Rykken et al. (18) demonstrated the usefulness of PRS in gadolinium-enhanced T1w sequences for the 
differential diagnosis between intramedullary spinal cord metastases and primary solid gadolinium-enhancing lesions; an evaluation not yet performed on brain metastases so far. In our population, PRS was more frequently observed in CMs (26 out of 33) than in HGGs (9 out of 28), this difference being statistically significant at Fisher Exact Test.

Unlike in HGG, the brain areas surrounding CMs comprise predominantly vasogenic edema, without significant brain infiltration, and thus the lesion borders may appear more defined and inscribed in the gadoliniumenhancing area. This would explain the observation of PRS following gadolinium injection, while the central part of the lesion appears less enhancing. However, no histopathologic correlates of the peripheral rim sign have been identified, including no evidence for a tumor capsule (18). Moreover, the non-negligible evidence of PRS in HGG (32\% as the only sign, $21 \%$ with a concomitant SAAC sign) suggests that this may represent an imaging finding not related to a specific pathologic process.

The assessment of PRS and SAAC is a fast procedure, easily feasible in a good quality conventional MRI morphologic study. When systematically performed, this assessment would improve the possibility of differentiating CMs from HGGs, even when more sophisticated and advanced diagnostic MR techniques are not available.

Both T1w SPGR and fast FLAIR gadolinium-enhanced images are used in daily practice. The assessment of both readers was qualitative regarding the evidence of PRS and SAAC signs. In gadolinium-enhanced SPGR images, flow related artifacts have been found to be significantly reduced when compared to Spin Echo images $(22,23)$. Despite slightly increased imaging artifacts (which, however, do not interfere with image interpretation) and longer acquisition time, gadolinium-enhanced $\mathrm{T} 1 \mathrm{w}-$ FLAIR imaging provides good lesion conspicuity and overall image contrast (24-29). At 3T, various 3D MRI sequences are tested for the detection of brain metastases $(30,31)$.

While showing encouraging results, the present study is limited by its retrospective nature. A more robust validation of our approach would require a prospective analysis, involving a higher number of patients. Additionally, it may be useful to compare different gadolinium-enhanced $\mathrm{T} 1 \mathrm{w}$ sequences at both $1.5 \mathrm{~T}$ and $3 \mathrm{~T}$. Finally, as in the case of intramedullary spinal cord masses (18), future work should include dedicated correlation between neuroradiology, surgery, and histopathologic analysis of microscopic findings at the tumor margins. Neurosurgeons attempting to remove brain tumors encounter various degrees of resectability and it would be interesting to analyse whether the rim sign predicts the presence of a cleavage plane and, in turn, an easier, more complete resection.

\section{Conclusion}

In conclusion, our study suggests that, in case of a solitary, solid, gadolinium-enhancing lesion, the observation of SAAC is quite specific for HGG. The presence of SAAC is highly suggestive of HGG, even when in combination with PRS. When no SAAC is found, the presence of PRS is a more frequent and relatively specific sign for CMs than for HGGs.

\section{Conflicts of Interest}

The Authors declare that there is no conflict of interest.

\section{Authors' Contributions}

CFM conceived and designed the study and wrote the paper; ET contributed data tools and wrote the paper; LU and RC collected the data and performed the analysis; GE designed the study; FC participated to the design and coordination and helped to draft the manuscript.

\section{Acknowledgements}

Funding: This research received no specific grant from any funding agency in the public, commercial, or not-for-profit sectors.

\section{References}

1 Giese A and Westphal M: Treatment of malignant glioma: a problem beyond the margins of resection. J Cancer Res Clin Oncol 127: 217-225, 2001. PMID: 11315255.

2 Soffietti R, Rudā R and Mutani R: Management of brain metastases. J Neurol 249: 1357-1369, 2002. PMID: 12382150. DOI: $10.1007 / \mathrm{s} 00415-002-0870-6$.

3 Schellinger PD, Meinck HM and Thron A: Diagnostic accuracy of MRI compared to CCT in patients with brain metastases. J Neurooncol 44: 275-281, 1999. PMID: 10720207.

4 Barnard RO and Geddes JF: The incidence of multifocal cerebral gliomas. A histologic study of large hemisphere sections. Cancer 60: 1519-1531, 1987. PMID: 3113716.

5 Schiff D: Single brain metastasis. Curr Treat Options Neurol 3: 89-99, 2001. PMID: 11123862.

6 Young GS: Advanced MRI of Adult Brain Tumors. Neurol Clin 25: 947-973, 2007. PMID: 17964022. DOI:10.1016/j.ncl. 2007.07.010.

7 Law M, Cha S, Knopp EA, Johnson G, Arnett J and Litt AW: High-grade gliomas and solitary metastases: Differentiation by using perfusion and proton spectroscopic MR imaging. Radiology 222: 715-721, 2002. PMID: 11867790. DOI: 10.1148/ radiol.2223010558.

8 Bulakbasi N, Kocaoglu M, Farzaliyev A, Tayfun C, Ucoz T and Somuncu I: Assessment of diagnostic accuracy of perfusion MR imaging in primary and metastatic solitary malignant brain tumors. AJNR Am J Neuroradiol 26: 2187-2199, 2005. PMID: 16219821.

9 Young GS and Setayesh K: Spin-Echo echo-planar perfusion MR imaging in the differential diagnosis of solitary enhancing brain lesions: Distinguishing solitary metastases from primary glioma. Am J Neuroradiol 30: 575-577, 2009. PMID: 19095787. DOI: 10.3174/ajnr.A1239 
10 Wang S, Kim S, Chawla S, Wolf RL, Zhang W-G, O'Rourke DM, Judy KD, Melhem ER and Poptani H: Differentiation between glioblastomas and solitary brain metastases using diffusion tensor imaging. Neuroimage 44: 653-660, 2009. PMID: 21330399. DOI: 10.3174/ajnr.A2333

11 Scarabino T, Popolizio T, Trojsi F, Giannatempo G, Pollice S, Maggialetti N, Carriero A, Di Costanzo A, Tedeschi G and Salvolini U: Role of advanced MR imaging modalities in diagnosing cerebral gliomas. Radiol Med 114: 448-460, 2009. PMID: 19082784. DOI: 10.1007/s11547-008-0351-9

12 Abdel Razek AAK, El-Serougy L, Abdelsalam M, Gaballa G and Talaat M: Differentiation of primary central nervous system lymphoma from glioblastoma: quantitative analysis using arterial spin labeling and diffusion tensor imaging. World Neurosurg 123: e303-e309, 2019. PMID: 30502475. DOI: 10.1016/ j.wneu.2018.11.155

13 El-Serougy L, Abdel Razek AAK, Ezzat A, Eldawoody H and El-Morsy A: Assessment of diffusion tensor imaging metrics in differentiating low-grade from high-grade gliomas. Neuroradiol J 29: 400-407, 2016. PMID: 27562582. DOI: 10.1177/19714 00916665382

14 Razek AAKA, El-Serougy L, Abdelsalam M, Gaballa G and Talaat M: Differentiation of residual/recurrent gliomas from postradiation necrosis with arterial spin labeling and diffusion tensor magnetic resonance imaging-derived metrics. Neuroradiology 60: 169-177, 2018. PMID: 29218370. DOI: 10.1007/s00234-017-1955-3

15 Cocozza S, Russo C, Pontillo G, Ugga L, Macera A, Cervo A, De Liso M, Di Paolo N, Ginocchio MI, Giordano F, Leone G, Rusconi G, Stanzione A, Briganti F, Quarantelli M, Caranci F, D'Amico A, Elefante A, Tedeschi E and Brunetti A: Is advanced neuroimaging for neuroradiologists? A systematic review of the scientific literature of the last decade. Neuroradiology 58, 2016. PMID: 27826667. DOI: 10.1007/s00234-016-1761-3

16 Caranci F, Tedeschi E, Leone G, Reginelli A, Gatta G, Pinto A, Squillaci E, Briganti $\mathrm{F}$ and Brunese L: Errors in neuroradiology. Radiol Med 120: 795-801, 2015. PMID: 26183038. DOI: 10.1007/s11547-015-0564-7

17 Kelly PJ, Daumas-Duport C, Kispert DB, Kall BA, Scheithauer BW and Illig JJ: Imaging-based stereotaxic serial biopsies in untreated intracranial glial neoplasms. J Neurosurg 66: 865-874, 1987. PMID: 3033172. DOI: 10.3171/jns.1987.66.6.0865

18 Rykken JB, Diehn FE, Hunt CH, Eckel LJ, Schwartz KM, Kaufmann TJ, Wald JT, Giannini C and Wood CP: Rim and flame signs: Postgadolinium MRI findings specific for non-CNS intramedullary spinal cord metastases. Am J Neuroradiol 34: 908-915, 2013. PMID: 23079405. DOI: 10.3174/ajnr.A3292

19 Tang YM, Ngai S and Stuckey S: The solitary enhancing cerebral lesion: can FLAIR aid the differentiation between glioma and metastasis? AJNR Am J Neuroradiol 27: 609-611, 2006. PMID: 16552003.

20 Muccio CF, Tarantino A, Esposito G and Cerase A: Differential diagnosis by unenhanced FLAIR T2-weighted magnetic resonance images between solitary high grade gliomas and cerebral metastases appearing as contrast-enhancing corticosubcortical lesions. J Neurooncol 103: 713-717, 2011. PMID: 21069426. DOI: $10.1007 / \mathrm{s} 11060-010-0454-8$
21 Zhang $\mathrm{M}$ and Olsson Y: Hematogenous metastases of the human brain--characteristics of peritumoral brain changes: a review. J Neurooncol 35: 81-89, 1997. PMID: 9266444.

22 Shogry MEC and Elster AD: Cerebrovascular enhancement in spoiled GRASS (SPGR) Images. J Comput Assist Tomogr 16: 48-53, 1992. PMID: 1729306.

23 Pui MH and Fok EC: MR imaging of the brain: comparison of gradient-echo and spin-echo pulse sequences. Am J Roentgenol 165: 959-962, 1995. PMID: 7677001. DOI: 10.2214/ ajr.165.4.7677001

24 Al-Saeed O, Ismail M, Athyal R, Rudwan M and Khafajee S: T1-weighted fluid-attenuated inversion recovery and T1weighted fast spin-echo contrast-enhanced imaging: A comparison in 20 patients with brain lesions. J Med Imaging Radiat Oncol 53: 366-372, 2009. PMID: 19695043. DOI: 10.1111/j.1754-9485.2009.02093.x

25 Lee JK, Choi HY, Lee SW, Baek SY and Kim HY: Usefulness of T1-weighted image with fast inversion recovery technique in intracranial lesions: comparison with T1-weighted spin echo image. Clin Imaging 24: 263-269, 2000. PMID: 11331152.

26 Tomura N, Narita K, Takahashi S, Otani T, Sakuma I, Yasuda K, Nishii $\mathrm{T}$ and Watarai J: Contrast-enhanced multi-shot echoplanar FLAIR in the depiction of metastatic tumors of the brain: comparison with contrast-enhanced spin-echo T1-weighted imaging. Acta Radiol 48: 1032-1037, 2007. PMID: 17957522. DOI: $10.1080 / 02841850701499425$

27 Caranci F: Perfusion and spectroscopy magnetic resonance imaging in a case of lymphocytic vasculitis mimicking brain tumor. Polish J Radiol 78: 66-69, 2013. PMID: 24115963. DOI: 10.12659/PJR.884011

28 Brunetti A, Alfano B, Soricelli A, Tedeschi E, Mainolfi C, Covelli EM, Aloj L, Panico MR, Bazzicalupo L and Salvatore M: Functional characterization of brain tumors: An overview of the potential clinical value. Nucl Med Biol 23: 699-715, 1996. PMID: 8940713.

29 Chen W, Wang L, Zhu W, Xia L, Qi J, Feng D and Luo X: Multicontrast single-slab 3D MRI to detect cerebral metastasis. Am J Roentgenol 198: 27-32, 2012. PMID: 22194476. DOI: 10.2214/AJR.11.7030

30 Schettino C, Caranci F, Lus G, Signoriello E, Eoli M, Anghileri E, Pollo B, Melone MAB, Di Iorio G, Finocchiaro G, Ugga L and Tedeschi E: Diffuse glioblastoma resembling acute hemorrhagic leukoencephalitis. Quant Imaging Med Surg 7: 592597, 2017. PMID: 29184769. DOI: 10.21037/qims.2017.06.09

31 Yoshida A, Tha KK, Fujima N, Zaitsu Y, Yoshida D, Tsukahara A, Onodera S, Shirato H and Terae S: Detection of brain metastases by 3-dimensional magnetic resonance imaging at 3 T. J Comput Assist Tomogr 37: 84-90, 2013. PMID: 23321838. DOI: $10.1097 /$ RCT.0b013e318271f216
Received July 15, 2019

Revised July 19, 2019

Accepted July 22, 2019 